\title{
Electrocardiogram Reference Range Indicator
}

National Cancer Institute

\section{Source}

National Cancer Institute. Electrocardiogram Reference Range Indicator. NCI Thesaurus.

Code C83263.

A indication that electrocardiogram test data lies within specified parameters. 